\title{
Palladium on Layered Double Hydroxide: A Heterogeneous System for the Enol Phosphate Carbon-Oxygen Bond Activation in Aqueous Media
}

\author{
Jaqueline D. Senra, ${ }^{1}$ Aires C. Silva, ${ }^{2}$ Raquel V. Santos, ${ }^{1}$ \\ Luiz Fernando B. Malta, ${ }^{2}$ and Alessandro B. C. Simas ${ }^{3}$ \\ ${ }^{1}$ Instituto de Química, Universidade do Estado do Rio de Janeiro, 20550-900 Rio de Janeiro, RJ, Brazil \\ ${ }^{2}$ Instituto de Química, Universidade Federal do Rio de Janeiro, 21941-903 Rio de Janeiro, RJ, Brazil \\ ${ }^{3}$ Instituto de Pesquisas de Produtos Naturais Walter B. Mors, Universidade Federal do Rio de Janeiro, \\ 21941-614 Rio de Janeiro, RJ, Brazil
}

Correspondence should be addressed to Jaqueline D. Senra; jdsenra@gmail.com and Luiz Fernando B. Malta; lfbmalta@iq.ufrj.br Received 7 September 2016; Accepted 23 October 2016; Published 3 January 2017

Academic Editor: Bartolo Gabriele

Copyright (C) 2017 Jaqueline D. Senra et al. This is an open access article distributed under the Creative Commons Attribution License, which permits unrestricted use, distribution, and reproduction in any medium, provided the original work is properly cited.

In this work, a new catalytic approach for the C-O activation of enol phosphates based on a palladium supported on layered double hydroxide was developed. In this case, two different ketene aminal phosphates were used as models to study the synthesis of $\alpha$-phenyl enecarbamates N-Boc/CBz under the Suzuki-Miyaura conditions. The use of an ortho-bromoaniline as precursor allowed the synthesis of the 2-phenyl indole through an arylation/Heck cyclization. Catalyst reusability enabled the synthesis of the heterocycle in moderate yields for four consecutive runs.

\section{Introduction}

Carbon-oxygen bonds have ubiquitous presence in several major natural and synthetic products. Among some chemical properties that contribute to their occurrence in complex systems the relative inertness of the $\mathrm{C}-\mathrm{O}$ bond can be considered crucial, since the bond dissociation energy is around 60 to $110 \mathrm{kcal} / \mathrm{mol}$ depending on the functionality $[1,2]$. However, recent progress in the development of metal catalysts containing specific active sites has drawn the attention of chemists to the widely existing $\mathrm{O}$-containing organic compounds in virtue of their low cost as organic electrophiles. In the context of metal-catalyzed cross-coupling reactions, the use of more reliable coupling partners is desired for both economical and ecological reasons compared to the traditional halides [24]. Of these, some most widely exploited in synthesis are the triflates due to their high reactivity compared to aryl/alkenyl halides $[5,6]$. Apart from this, there are several disadvantages concerning the use of triflates, including their high cost and some difficulties in the handling. On the other hand, the widely existing O-electrophiles such as phenols, alcohols, ethers, and esters have more recently been considered as promising starting platforms in large-scale processes envisaging the use of biomass-derived products.

Phosphates represent one of the most important classes of esters since they have an essential role in nature $[7,8]$. In general, their leaving ability occurs mostly in the presence of enzyme catalysis so their suitable activation under normal conditions depends mainly on the nucleophile along with the $\mathrm{pH}$. Some palladium complexes have been known for their ability to catalyze the addition of carbon and nitrogen nucleophiles onto alkyl and allyl phosphates [9]. However, depending on the reaction conditions, a complex end mixture consisting of the $\mathrm{S}_{\mathrm{N}} 2$ and $\mathrm{S}_{\mathrm{N}} 2^{\prime}$ products can be obtained instead of the metal-catalyzed alkylation/arylation. In contrast to other enolate derivatives, enol phosphates generally have an improved stability, which avoids the formation of side products and also the production of undesirable waste material during the synthesis [10]. 
There have been a number of synthetic applications involving the use of enol phosphates and some correlates (e.g., phosphonates and phosphonites) as starting materials for the conversion into a variety of functional groups [2, 1117]. In these cases, enol phosphates have mostly been useful substrates for some metal-catalyzed cross-coupling reactions, such as Suzuki-Miyaura, Sonogashira, Stille, Negishi, and Kumada mostly by palladium(0) and nickel( 0 ) species. The earlier reports involved the seminal studies by Takai and coworkers which started exploring the alkylation of enol phosphates with trialkylaluminium [11]. By considering strategies involving both $\mathrm{Csp}^{2}$ coupling partners, some interesting applications by the Nicolaou et al. [12] and Fuwa [16] groups have been recently described towards the total synthesis of natural products. Among some of these applications, ketene aminal phosphates ( $\alpha$-phosphoryloxy enecarbamate) were found to be specially convenient for the construction of complex nitrogen-containing heterocycles by exploring the palladium catalysis [18-20]. However, some limitations are related to the use of specific and expensive ligands along with the relatively high amounts of catalyst.

Recently, the interest in cutting-edge heterogeneous materials exhibiting high activity for multistep synthesis has led to the emergence of different classes of catalysts with unique properties. Layered double hydroxides (LDHs) are well-known two-dimensional (2D) anionic clay materials having a brucite-like layers and formula $\left[\mathrm{M}^{\mathrm{II}}(1-x), \mathrm{M}^{\mathrm{III}}{ }_{x}(\mathrm{OH})_{2}\right]\left[\mathrm{A}^{n-}(x / n)\left(\mathrm{H}_{2} \mathrm{O}\right)\right]$. Due to relatively weak interlayer bonding a wide tunability to compensating anions results in materials with compositional flexibility and interesting applications in catalysis, especially under basic conditions [21]. For instance, LDHs containing transitionmetals intercalated in the layers or metal nanoparticles have been used as efficient catalysts for cross-couplings with aryl halides. In most cases, the layered matrix was exploited as special supports since high turnover frequencies (TOFs) were observed under relatively mild reaction conditions. Recently, our research group demonstrated that LDHs of $\mathrm{Mg} / \mathrm{Al}$ with different crystallinities can behave distinctly regarding the ability for intercalation of palladium or gold species [22, 23]. The application of the Pd catalyst system in the Suzuki-Miyaura reaction with aryl bromides enabled high yields for the corresponding biaryls under neat water and room temperature [23]. As far as we know, the exploration of oxygen-based electrophiles for carbon-carbon bond formation under heterogeneous catalysis is scarce. Here, we present first results concerning the enol phosphate (ketene aminal phosphate) C-O bond activation promoted by a LDH system containing palladium.

\section{Experimental}

All reactions were performed under air using conventional reflux glassware. All chemicals were purchased at the highest commercial grade and used as received. Analytical thin-layer chromatography (TLC) was performed by using E. Merck silica gel 60 F254 precoated glass plates ( $0.25 \mathrm{~mm}$ thickness). Flash column chromatography was carried out by using E. Merck silica gel BW-300 (200-400 mesh).
Fourier-Transform Infrared Spectroscopy. The measurements were performed on Nicolet Magna 760 spectrophotometer with 16 accumulations and $4 \mathrm{~cm}^{-1}$ resolution in the 4000 $400 \mathrm{~cm}^{-1}$ region. The samples were prepared in $\mathrm{KBr}$ pellets.

$X$-Ray Diffraction. The X-ray diffractograms were acquired at ambient temperature with a Rigaku diffractometer, Ultima IV model with high frequency X-ray generator $(3 \mathrm{~kW}), \mathrm{Cu} \mathrm{X}$ ray tube $(\lambda=1.5418 \AA \AA$ ) $)$, Universal Theta-2 Theta goniometer with $185 \mathrm{~mm}$ radius, fixed slits, and Ni Kbeta filter with $40 \mathrm{kV}$ voltage and $20 \mathrm{~mA}$ current. Samples were analyzed on glass supports. The measurements conditions were $5<2 \theta<80$ degrees, $0.05^{\circ}$ step, and a step time of 1 second.

$X$-Ray Fluorescence. The compounds were analyzed on a Philips spectrophotometer, model PW 2400 with rhodium lamp. Samples were fused with lithium tetraborate for the formation of pellets.

Scanning Electron Microscopy and Energy-Dispersive X-Ray Spectroscopy. The photomicrographs of scanning electron microscopy (SEM) were acquired by a JEOL JSM 6460$\mathrm{LV}$, operating between 10 and $20 \mathrm{kV}$ equipped with energy dispersive X-ray spectrometer to perform the chemical microanalysis. The powders were adhered to a double-sided tape graphite attached to the sample holder. Some samples were metallized with gold for better viewing. The elemental analysis via energy dispersive X-ray spectroscopy (EDS) had the following parameters: resolution: $256 \times 192$; cutting time: $50 \mathrm{~s}$; number of cuts: 5; acquisition time: $250 \mathrm{~s}$; residence time: $712 \mu \mathrm{s}$.

BET Analysis. Nitrogen adsorption/desorption isotherms were obtained at $-196^{\circ} \mathrm{C}$ using the instrument Quantachrome Novawin. The samples were pretreated in vacuum at $180^{\circ} \mathrm{C}$ for 5 hours. The specific surface area of the materials was calculated by the BET method [24].

${ }^{1} \mathrm{H}$ NMR Analysis. ${ }^{1} \mathrm{H}$ NMR spectra of products $\mathbf{2 a}, \mathbf{2} \mathbf{b}$, $\mathbf{3 a}-\mathbf{3} \mathbf{c}$, and $\mathbf{4 a}-\mathbf{4} \mathbf{c}$ are presented in the Supplementary Information (see Supplementary Material available online at https://doi.org/10.1155/2017/8418939). ${ }^{1}$ H NMR spectra were recorded on a Varian VNMRSYS-500 spectrometer at $25^{\circ} \mathrm{C}$. Chemical shift values are reported in $\delta$ (ppm) relative to tetramethylsilane with reference to internal residual solvent. Coupling constants $(J)$ are reported in hertz $(\mathrm{Hz})$. Multiplicities of signals are designated as follows: $\mathrm{s}=$ singlet; $\mathrm{d}=$ doublet; $\mathrm{t}=$ triplet; $\mathrm{m}=$ multiplet; $\mathrm{br}=$ broad.

\subsection{TH-LDH Synthesis Containing Pd}

2.1.1. Synthesis of the Hydrothermally Treated Layered Double Hydroxide. In a $250 \mathrm{~mL}$ round-bottomed flask with milli$\mathrm{Q}$ water $(100 \mathrm{~mL}), \mathrm{Mg}\left(\mathrm{NO}_{3}\right)_{2} \cdot 6 \mathrm{H}_{2} \mathrm{O}(0.006 \mathrm{~mol}, 1.538 \mathrm{~g})$ and $\mathrm{Al}\left(\mathrm{NO}_{3}\right)_{3} \cdot 9 \mathrm{H}_{2} \mathrm{O}(0.003 \mathrm{~mol}, 1.125 \mathrm{~g})$ were added with a $\mathrm{Mg} / \mathrm{Al}$ molar ratio of 2. Furthermore, under strong magnetic stirring $\mathrm{NaOH}$ aqueous solution $\left(1 \mathrm{~mol} \mathrm{~L}^{-1}\right)$ was added dropwise until a final $\mathrm{pH}$ around 10. After the coprecipitation the material obtained was hydrothermally treated $\left(80^{\circ} \mathrm{C}\right)$ for 24 hours and 
the material was filtered via common filtration and dried at room temperature.

2.1.2. Immobilization of Pd on Layered Double Hydroxide. The immobilization was processed using $0.025 \mathrm{~mol} \mathrm{~L}^{-1}$ solution<smiles>CC(=O)N(C(C)=O)c1ccccc1Br</smiles>

(3c) of $\mathrm{Na}_{2} \mathrm{PdCl}_{4}$ in $\mathrm{N}, \mathrm{N}$-dimethylformamide (DMF) applying the molar ratio $\mathrm{Pd}: \mathrm{HDL}$ of $1: 2$ at $80^{\circ} \mathrm{C}$ for 24 hours.

\subsection{Typical Procedure for the Synthesis of Enol Phosphate 4c}

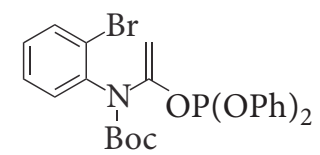

(4c)
A solution of BuLi 1.04 $\mathrm{M}$ in hexanes $(3.0 \mathrm{~mL})$ was added to i- $\mathrm{Pr}_{2} \mathrm{NH}(0.48 \mathrm{~mL}, 3.5 \mathrm{mmols})$ in dry THF $(8 \mathrm{~mL})$ under Ar at $0^{\circ} \mathrm{C}$. After $30 \mathrm{~min}$, imide $3 \mathrm{c}(0.384 \mathrm{~g}, 1.60 \mathrm{mmols})$ in dry THF $(1.5 \mathrm{~mL})$ was added at $-78^{\circ} \mathrm{C}$ and the mixture was stirred for $1 \mathrm{~h}$ under the same conditions. Then, a solution of $(\mathrm{PhO})_{2} \mathrm{POCl}(0.65 \mathrm{~mL}, 3.12 \mathrm{mmols})$ in dry THF $(2.3 \mathrm{~mL})$ was added dropwise and stirring proceeded for $1 \mathrm{~h}$. Then, $5 \%$ aq $\mathrm{NH}_{4} \mathrm{OH}$ was added and after regular work-up, the obtained residue was purified by flash chromatography (neutralized silica gel, 2\% TEA) to afford phosphate $4 \mathrm{c}(0.65 \mathrm{~g}, 78 \%)$.

\subsection{Typical Procedure for the Synthesis of Product $\mathbf{8}$}

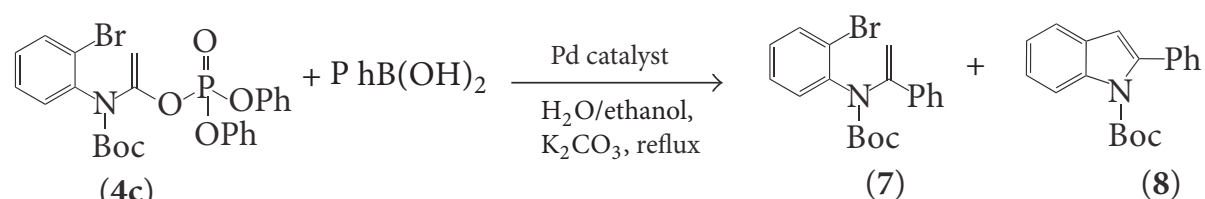

A mixture of enol phosphate $4 \mathrm{c}(0.116 \mathrm{~g}, 0.218 \mathrm{mmol})$, phenylboronic acid $(0.164 \mathrm{~g}, 0.437 \mathrm{mmol})$, potassium carbonate $(0.028 \mathrm{~g}, 0.656 \mathrm{mmol})$, and HT-DLH $(0.012 \mathrm{~g}, 0.011 \mathrm{mmol})$ in water/ethanol $(2: 1,5.0 \mathrm{~mL})$ was refluxed for $24 \mathrm{~h}$. After this period, the reaction mixture was cooled, filtered, and extracted with a mixture of ethyl acetate/dichloromethane $(1: 1,3 \times 15 \mathrm{~mL})$. The combined organic layers were dried with $\mathrm{Na}_{2} \mathrm{SO}_{4}$ and after filtration/evaporation of the solvent, the residue was analyzed by $\mathrm{NMR}{ }^{1} \mathrm{H}$.

\section{Results and Discussion}

3.1. Characterization of the Hydrothermally Synthesized LDH Catalyst. Our first studies concerning the synthesis and characterization of the coprecipitated $\mathrm{LDH}$ evidenced that $\operatorname{Pd}(0)$ was formed during the ion-exchange process mediated by $N, N$-dimethylformamide (DMF) [23]. For this material, it was evidenced that $\operatorname{Pd}(0)$ was formed during the ionexchange process mediated by $N, N$-dimethylformamide. We inferred that the elementary species of palladium were inserted in the interlayer region [23]. In the following, we report the characterization of the palladium containing $\mathrm{LDH}$ whose matrix was submitted to hydrothermal treatment at $80^{\circ} \mathrm{C}$ for 24 hours.

Firstly data from X-rays fluorescence showed the incorporation of $0.5 \%$ palladium. It is worth noting that this LDH absorbs less palladium, approximately half the quantity, than coprecipitated LDH does (1.0\%) [23]. Concerning this we may conclude that the crystallinity of the matrix was influenced in the incorporation of palladium, probably due to the different kinds of species that were absorbed.
To proceed to a phase analysis in the LDH matrix we obtained the X-rays diffraction patterns (Figure 1). Figure 1(a) evidences that the hydrothermally treated LDH (HT-LDH) is more crystalline than the coprecipitated one [23], as expected. There was no shift of the (003) reflection ( $d=7.66$ angstroms) due to the incorporation of palladium in the matrix (Figure 1(b)). The same behavior was observed for the incorporation of Pd by the coprecipitated LDH [23]. In contrast the XRD pattern for the Pd containing HT-LDH, Figure $1(\mathrm{~b})$, presents no apparent $\operatorname{Pd}(0)$ peaks, which let us conclude that mostly $\mathrm{Pd}^{2+}$ species were incorporated in the matrix. However up to this point we cannot affirm that $\mathrm{PdCl}_{4}{ }^{2-}$ species were intercalated in the HT-LDH material.

In order to gain more information concerning the $\mathrm{Pd}$ incorporation process, Fourier-transform infrared (FTIR) spectra were acquired. These spectra are shown in Figure 2 and evidence that few differences are noticed when they are compared. Basically vibrational modes corresponding to $\nu_{\text {as }} \mathrm{CO}_{3}{ }^{2-}$ at $1369 \mathrm{~cm}^{-1}, \nu_{\text {as }} \mathrm{NO}_{3}{ }^{-}$at $1384 \mathrm{~cm}^{-1}$, and $\nu \mathrm{OH}$ relative to the interaction between interlayer $\mathrm{H}_{2} \mathrm{O}$ and $\mathrm{CO}_{3}{ }^{2-}$ at $3080 \mathrm{~cm}^{-1}$ [25] were kept for Pd/HT-HDL. Also the bands in the 1000-400 $\mathrm{cm}^{-1}$ range, relative to $\delta_{\mathrm{as}} \mathrm{CO}_{3}{ }^{2-}\left(640 \mathrm{~cm}^{-1}\right)$ and relative to $\mathrm{Al}-\mathrm{O}$ and $\mathrm{Al}-\mathrm{OH}$ modes (780, 550, and $450 \mathrm{~cm}^{-1}$ ) [26], remained unchanged in contrast of what was previously reported for the intercalation of noble metals by $\mathrm{LDH}[25,26]$. With these results in mind it may be said that most of the chemical conditions in the interlayer region seem to be unchanged after Pd incorporation.

To further investigate this chemical system, we managed to make some visualizations of scanning electron microscopy 


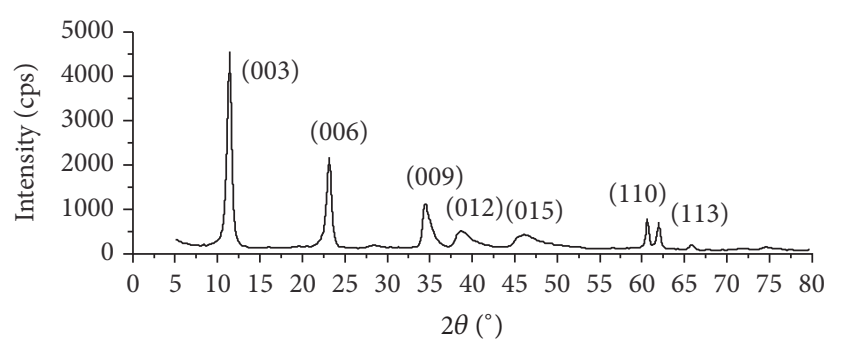

(a)

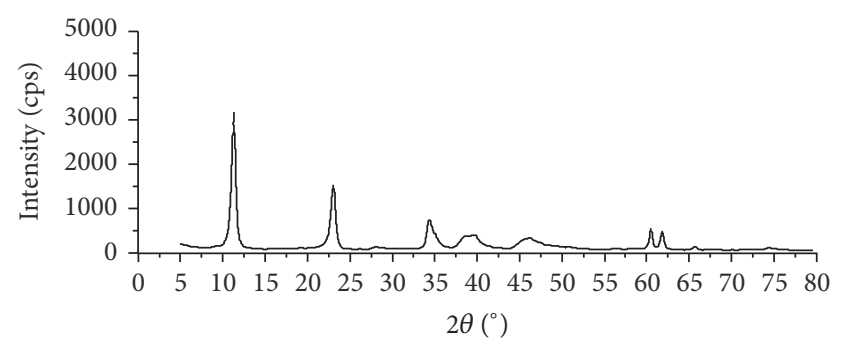

(b)

FIGURE 1: XRD patterns for the hydrothermally treated layered double hydroxide (HT-LDH) before (a) and after (b) palladium incorporation.

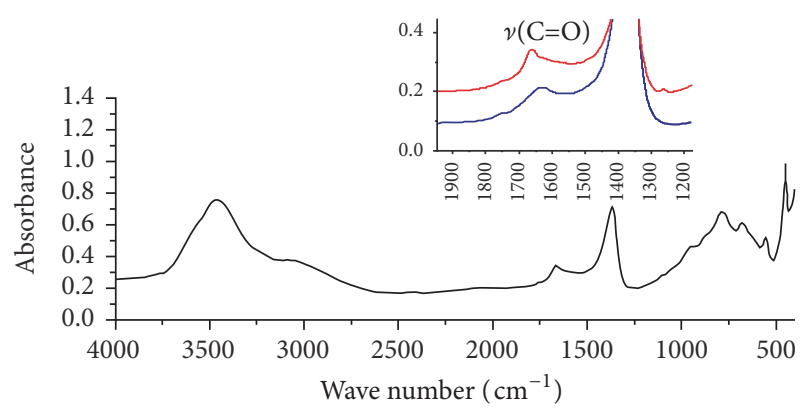

(b)

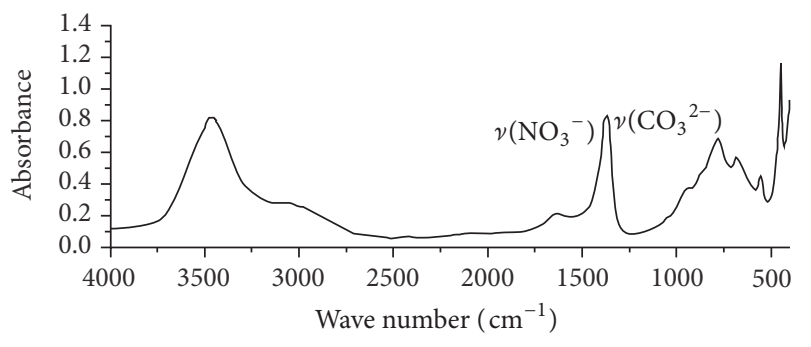

(a)

FIGURE 2: Fourier-transform infrared spectra for the hydrothermally treated layered double hydroxide (HT-LDH) before (a) and after (b) palladium incorporation. Inset: zoom of the 1900$1200 \mathrm{~cm}^{-1}$ region of both spectra signaling in the upper spectrum the band relative to $\mathrm{C}=\mathrm{O}$ stretching mode of $N, N$-dimethylformamide.

(Figure 3). From Figure 3(a), relative to a backscattered electrons image, a phase contrast evidenced by the white brilliant particles situated on the surface of the dark grey agglomerate was noticed. This is unique since this phase contrast was not observed for the coprecipitated LDH after Pd incorporation. We also obtained the elements distributions ( $\mathrm{Pd}, \mathrm{Cl}$, and $\mathrm{Mg}$ ) throughout this agglomerate by using the energy-dispersive $\mathrm{X}$-rays spectroscopy facility coupled to the SEM microscope (Figures 3(b)-3(d)). From the EDX element mapping it became clear that the brilliant phase corresponded to a higher density of the palladium and chlorine elements contrasting to a lower density of the magnesium element: rather than being caused by chemical inhomogeneity it was due to shielding of the Mg characteristic X-rays promoted by the superficial phase. This phase can be assigned as being composed of Pd and $\mathrm{Cl}$; however we do not believe that this would be the precursor salt $\mathrm{Na}_{2} \mathrm{PdCl}_{4}$, since it is very soluble in DMF. It is more probable to correspond to some palladium chloride that precipitated from the medium due to the association with DMF. In the IR spectrum of this catalyst we zoomed in the region of $1900-1200 \mathrm{~cm}^{-1}$ and we evidenced one band above $1650 \mathrm{~cm}^{-1}$ that can be assigned as the DMF carbonyl stretching mode (see the inset of Figure 2 for the comparison of this material IR spectrum with that of $\mathrm{LDH}$ before $\mathrm{Pd}$ incorporation). Therefore although part of $\mathrm{Pd}$ and $\mathrm{Cl}$ appeared homogeneously distributed throughout the particle in Figure 3(a), probably intercalated, another part composed a second phase that precipitated over LDH particles.

The nitrogen adsorption/desorption isotherms for the $\mathrm{Pd} / \mathrm{HT}-\mathrm{LDH}$ are shown in Figure 4(a). In this isotherm it is important to note how the hysteresis loop is narrow, which is characteristic of crystalline materials. This isotherm differs entirely from materials with low crystallinity as the Pd immobilized in coprecipitated LDH, Pd/CP-LDH (Figure 4(b)). In addition the surface area of the hydrothermally treated catalyst $\left(65.12 \mathrm{~m}^{2} \mathrm{~g}^{-1}\right)$ is also higher than that of the coprecipitated one $\left(7.834 \mathrm{~m}^{2} \mathrm{~g}^{-1}\right)$. With a hydrothermal treatment step, greater crystallites that have less reactive surface are generated and consequently agglomerate less. Smaller agglomerates correspond to smaller particles with higher surface areas therefore reasoning why the surface area of the hydrothermally treated $\mathrm{LDH}$ is higher compared to the coprecipitated one.

3.2. Reactions. Although several synthetic methodologies have been developed to access ketene aminal phosphates, the most convenient strategy consists of the deprotonation of an enolisable ketone using a strong base, such as LDA, followed by reaction of the subsequent metal enolate with a phosphoryl chloride (Scheme 1). Also, the stability of the phosphates depends on the electronic effects imposed on the nitrogen moiety. Previously, our group reported that stable ketene aminal phosphate ( $\alpha$-phosphoryloxy enecarbamate) derived from $N, N$-diprotected acetamide could be prepared by using $p$-methoxyphenyl (PMP) and $\mathrm{CBz}$ as protecting groups at the nitrogen [20]. In this case, cross-couplings under classical conditions, including the use of phosphine and arsine as ligands, were carried out to access enynes and dienes in moderate to good yields [20].

To evaluate the catalytic activity of the Pd/HT-LDH, we firstly run experiments with the PMP substituted, $N-\mathrm{CBz}-$ protected ketene aminal phosphate $4 \mathbf{a}$ since this starting 


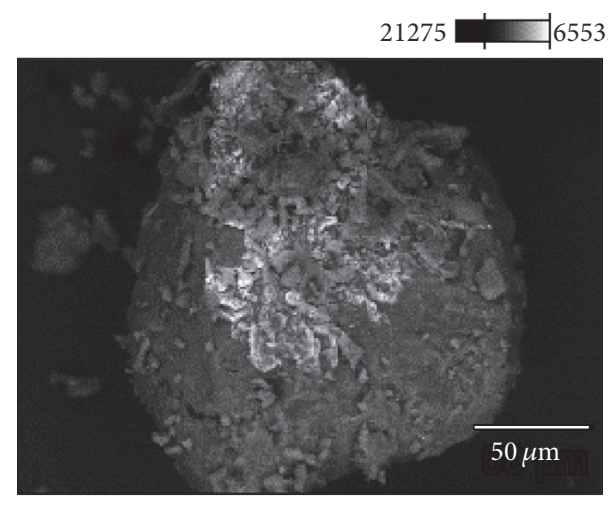

(a)

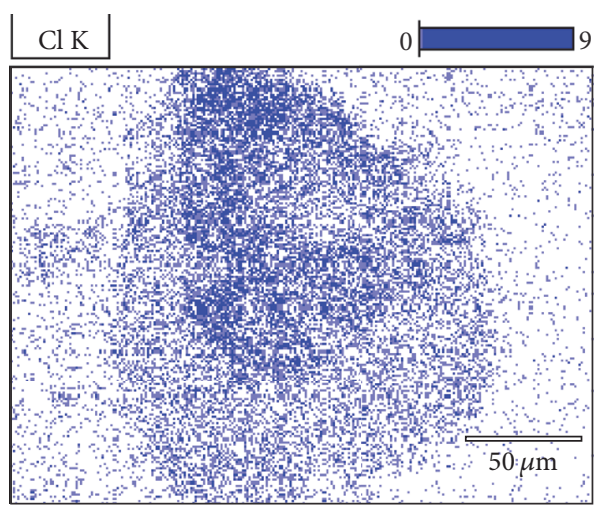

(c)

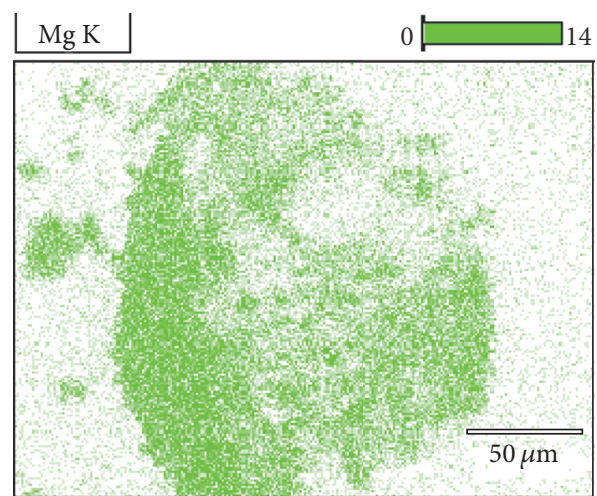

(b)

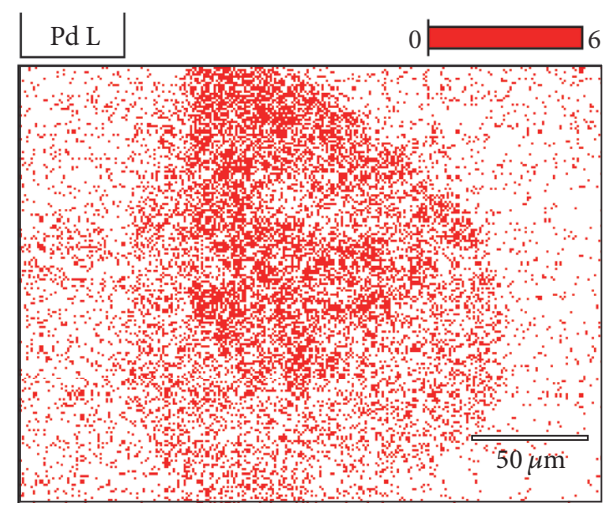

(d)

FIGURE 3: SEM-EDS visualizations for the catalyst of Pd on hydrothermally treated layered double hydroxide. (a) Backscattered electrons image; (b)-(d) EDS mapping of the elements $\mathrm{Mg}, \mathrm{Cl}$, and $\mathrm{Pd}$.

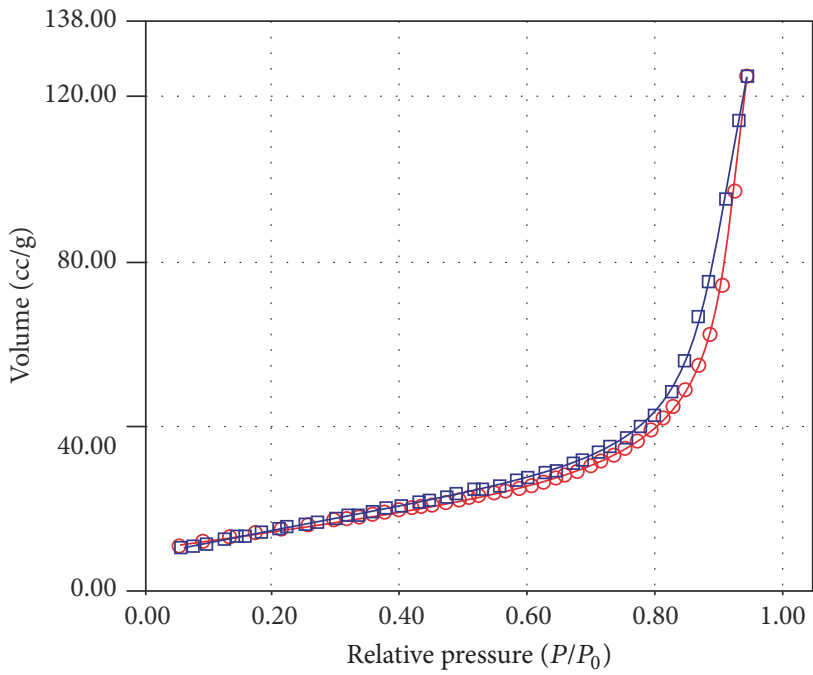

(a)

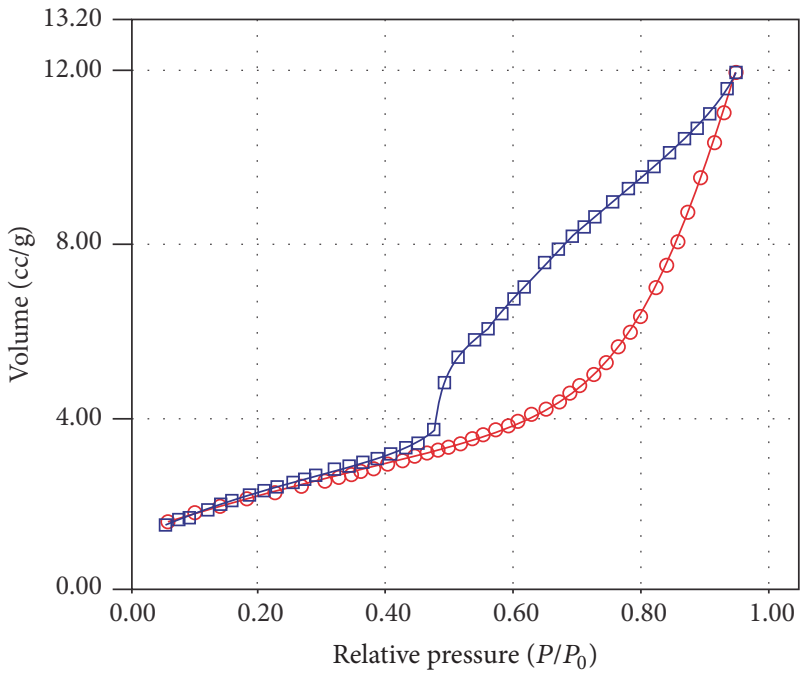

(b)

FIGURE 4: Nitrogen adsorption/desorption BET isotherms of the catalysts based on (a) hydrothermally treated and (b) coprecipitated layered double hydroxide. 


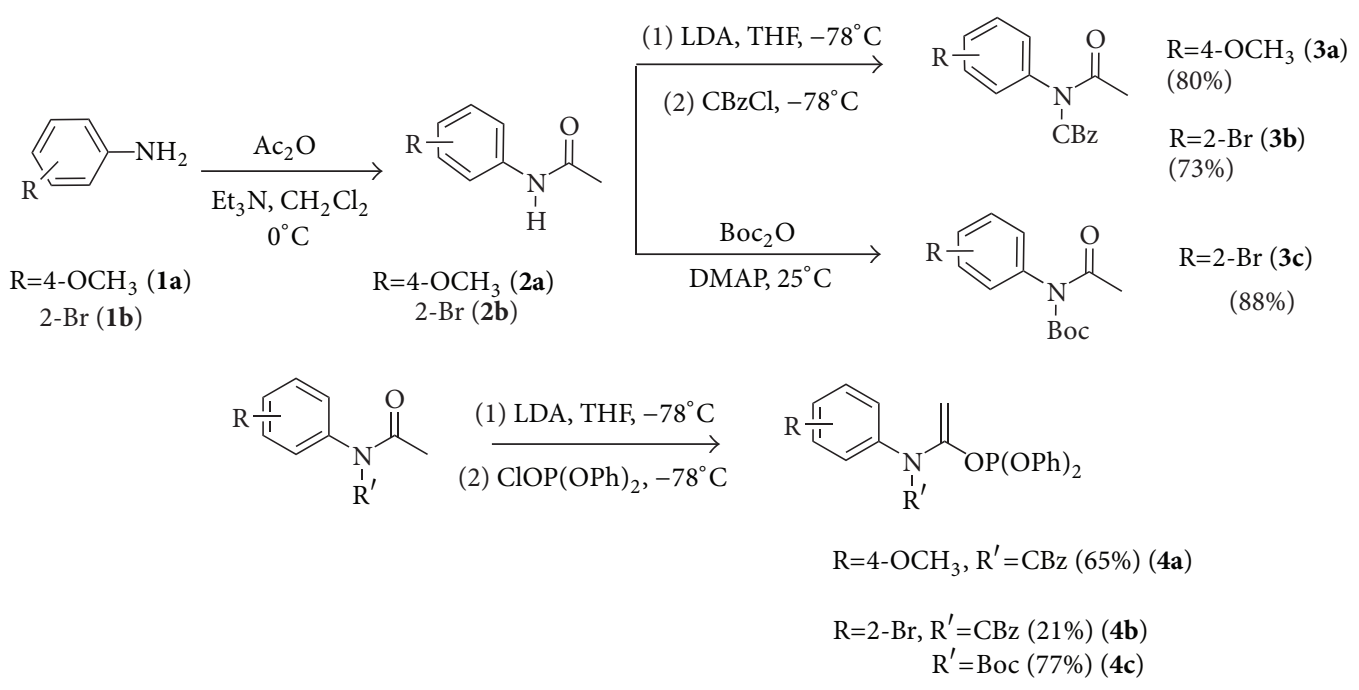

SCheme 1: Synthesis of the enol phosphates.

material exhibited adequate stability and could represent a model. Indeed, based on our previous work involving the catalytic evaluation of LDH in Suzuki-Miyaura reactions with aryl halides [22], we have explored the reaction with the phenylboronic acid by using some clean conditions. Since LDHs constitute a basic platform, the first catalytic test occurred in the absence of base but this condition did not afford the product (Table 1, entry 1). Thus, we explored the HT-LDH containing $1.0 \mathrm{~mol} \% \mathrm{Pd}, \mathrm{K}_{2} \mathrm{CO}_{3}$ as base and water/ethanol as solvent mixture under reflux $\left(85-90^{\circ} \mathrm{C}\right)$. However, the result indicated only a modest yield after $24 \mathrm{~h}$ (Table 1, entry 2). As expected, the increase on the Pd loading $(2.0 \mathrm{~mol} \%)$ led to the desired product in a slightly high yield (Table 1 , entry 3 ). Since the starting material solubility could be a reason for these relatively low conversions, we screened some different conditions. For instance, when it was carried out in neat water or ethanol only product traces were observed (Table 1, entries 4 and 5). However, the mixture composed of $\mathrm{H}_{2} \mathrm{O} / \mathrm{THF}(1: 1)$ was clearly superior, producing the desired coupled product $\mathbf{6 a}$ in $72 \%$ without the production of any side products (Table 1 , entry 6). It suggests that the solvent can have an additional influence in the reaction outcome.

An investigation of the two different $\mathrm{LDH}$ sevealed that the coprecipitated material has a poorer ability to catalyze the reaction under the same conditions (Table 1, entry 7), when compared to the hydrothermally treated one. This reactivity trend could be related to the presence of fewer active sites according to surface area analysis from BET isotherms. In order to gain further information concerning the use of a different ligand-free system, the PdNPs stabilized by hydroxypropylated cyclodextrins, earlier described by our group, were also tested under these conditions (Table 1, entry 8). It was apparent that a moderate conversion of 4a required about $5.0 \mathrm{~mol} \% \operatorname{Pd}(0)$ even though with some catalyst agglomeration. In this case, the use of high amounts of CDs was needed (Table 1, entry 9). On the other hand, the use of $\mathrm{Na}_{2} \mathrm{PdCl}_{4}$ as the only catalyst precursor proved to be ineffective for this reaction (Table 1, entry 10).

With the aim to test an alternative enol phosphate for consecutive Suzuki/Heck reactions, we proceeded to explore a substrate derived from an $o$-haloanilide (Table 1, entry 11) since it could be an elegant catalytic strategy for future synthetic exploration. However, under these conditions no conversion of $\mathbf{4 b}$ towards the expected coupling products was detected. Instead, it was evident by the $\mathrm{NMR}{ }^{1} \mathrm{H}$ analysis that the presence of some signals related to the substrate degradation suggesting a substrate lability. These experiments show that the $o$-brominated enol phosphate exhibited a behavior that is dramatically different from that of $\mathbf{4 a}$. With respect to the possible carbon-bromine bond activation it cannot be completely ruled out even though we could not found NMR signals related to this product. Unlike this referred cross-coupling we presume that the hydrodehalogenation has occurred. Then, once the carbon-metal bond has been formed, the challenge is to suppress the hydrogenolysis which produce the subproduct. Under the previous conditions, transmetallation and/or reductive elimination, therefore, must proceed more slowly than hydrogenolysis.

By virtue of the importance of the 2-substituted aryl indole structural motif [27-31], we have further investigated its synthetic feasibility by using this ligand-free catalytic system. In fact, there are two fundamental requirements for this challenging transformation: (1) the catalyst should exhibit preferential chemoselectivity towards the phosphate moiety to avoid the previous arylation of the halogenated one. (2) The usually disfavored 5-endo-trig Heck cyclization must have a higher reaction rate when compared to the hydrodehalogenation which can be a competitive side path. Indeed, it is necessary to take into account the subtle electronic effects promoted by the $N$-substituents since it can impose differences in the activation rate of each reaction site. Fuwa and coworkers $[18,32]$ have earlier explored this elegant strategy for the preparation of functionalized heterocycles 
TABLE 1: Screening of the reaction conditions for the synthesis of the $\alpha$-phenyl-enecarbamates from the $N$-CBz protected enol phosphates.

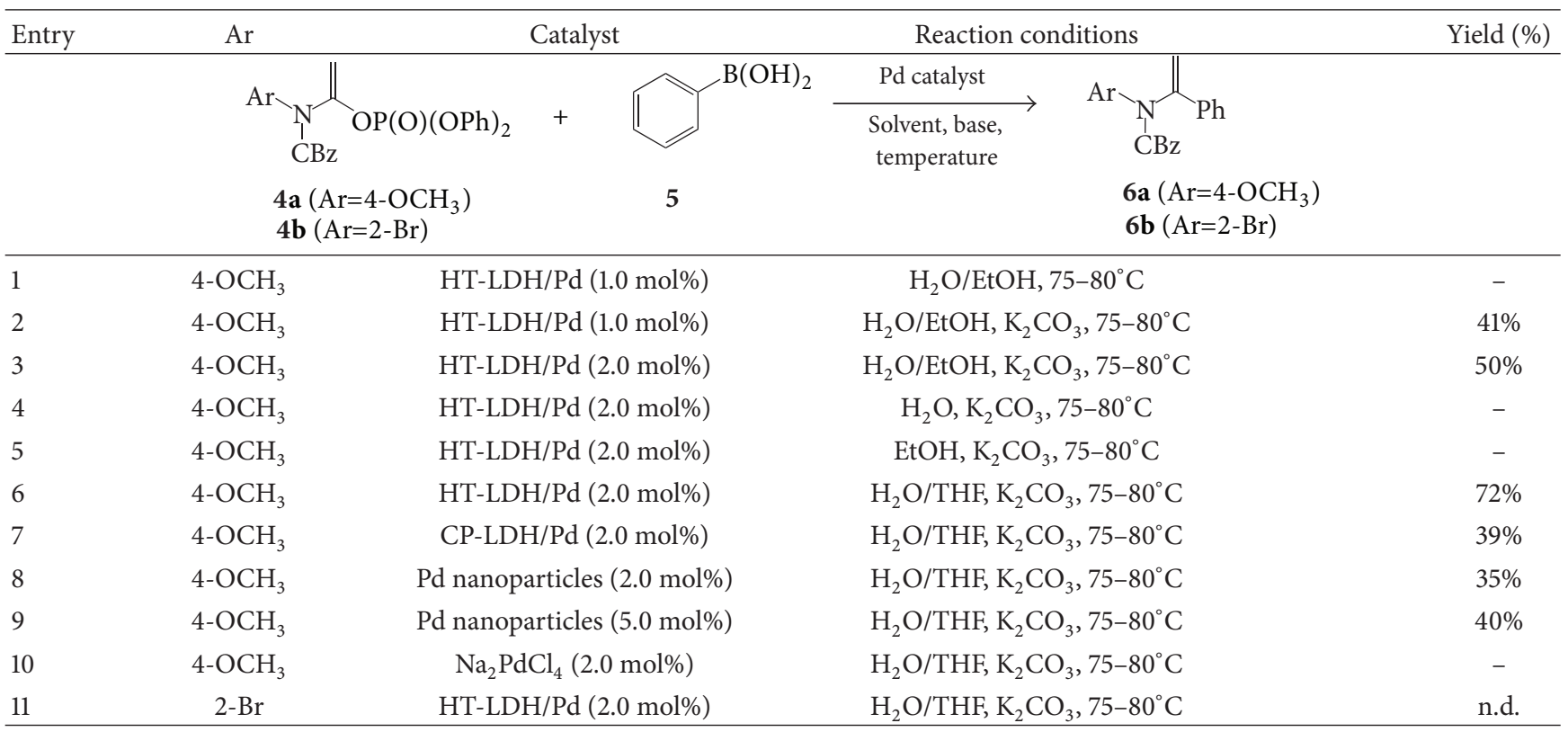

even by using the classical conditions $\left[\mathrm{Pd}\left(\mathrm{PPh}_{3}\right)_{4} 10 \mathrm{~mol} \%\right.$, $\mathrm{Et}_{3} \mathrm{~N}, \mathrm{DMF}, 100^{\circ} \mathrm{C}$, and sealed tube]. Starting from $o-$ bromoanilide, the corresponding halogenated ketene aminal phosphate was prepared for the catalytic tests. In this case, we have used the $N$-Boc-protected substrate, obtained in good yield (85\%), which presented a good stability when compared to the previous one. We presume that the absence of a strong donating group (PMP) improved the stability of the $\left(\mathrm{CH}_{3}\right)_{3} \mathrm{C}-\mathrm{O}$ bond in Boc moiety [20]. The examination of the Suzuki-Miyaura reaction between phenylboronic acid and substrate $4 \mathrm{c}$ in $\mathrm{H}_{2} \mathrm{O}$ : THF $(1: 1)$, in the presence of $2.0 \mathrm{~mol} \% \mathrm{Pd} / \mathrm{HT}-\mathrm{LDH}$ and $\mathrm{K}_{2} \mathrm{CO}_{3}$, revealed the formation of the $\alpha$-aryl enecarbamate 7 in $40 \%$ along with the minority cyclic product $8(17 \%)$ when the reaction was carried out by $24 \mathrm{~h}$ (Table 2, entry 1). However, running the reaction by $48 \mathrm{~h}$ did not improve the cyclization towards the indole ring but instead led to the partial degradation of 7 (Table 2, entry 2).

According to Fuwa and coworkers, the suppression of the cyclic product can occur with the addition of $\mathrm{H}_{2} \mathrm{O}$ and a moderate reaction temperature [32]. To evaluate if this finding follows a trend we performed a test in the presence of dry THF as the sole solvent. However, when compared to the previous condition $\left(\mathrm{H}_{2} \mathrm{O} / \mathrm{THF}\right)$ it gave an unsatisfactory result due to the incomplete starting material conversion and lack of selectivity (Table 2, entry 3). On the other hand, by using commercial ethanol, we have observed an almost complete consumption of the starting material as well as the formation of the cyclic product in a moderate yield (Table 2, entry 4). Based on this evidence, we argued that water could have a role in the formation of active species. Thus, our strategy involved the increase of the indole yield by using this heterogeneous catalytic system in the presence of a mixture based on water/ethanol. Under the condition involving water/ethanol $(2: 1)$, the Suzuki-Miyaura reaction tandem cyclization with the HT-LDH took place in the presence of $\mathrm{K}_{2} \mathrm{CO}_{3}$ at $90^{\circ} \mathrm{C}$ for $24 \mathrm{~h}$ affording an inversion in the $7: 8$ ratio with an improved yield for the indole (Table 2, entry 5 ). However, by changing the base to cesium carbonate the efficiency of the cyclization was affected (Table 2, entry 6).

It is interesting to note that the $7: \mathbf{8}$ ratio can be tuned not only by changing the solvent but also through the catalyst system: by using the $\mathrm{Pd}$ nanoparticles stabilized by CDs, the cyclization proceeded with a poor selectivity towards $\mathbf{8}$ (Table 2, entry 7). By comparison, the coprecipitated LDH was also tested under this condition to verify the influence of the catalyst structure on the cyclization. As shown in entry 8 , the reaction occurred with similar selectivity suggesting that it can be improved by the LDH structural effect involving the interlayer space.

Considering the formation of $\mathbf{7}$ and $\mathbf{8}$, we found, in a control experiment, that the hypothetical product from intramolecular Heck reaction (indole formation) containing the phosphate moiety is not involved as the first intermediate since no reaction occurred in the absence of the phenylboronic acid. This data supports the idea of a mechanism in which the $\mathrm{C}-\mathrm{O}$ bond activation occurs followed by the carbon-halogen coupling. In addition the catalyst deactivation could be a hypothesis that for prolonged times the reaction does not afford the cyclization leading to indole. However its recycling ability was showed (see Table 3 ) hence contrasting to the hypothesis of its deactivation. Any poisoning of the LDH by inspection of the catalyst without washing/purification using infrared spectroscopy was not detected.

To test the merit concerning the nature of the catalysis, we performed in a separate experiment a hot filtration test as a useful criterion for distinguishing between homogeneous and heterogeneous catalysis [33]. In this case, the reaction 
TABLE 2: Screening of the reaction conditions for the synthesis of the 2-phenyl indole from the $N$-Boc protected enol phosphate.

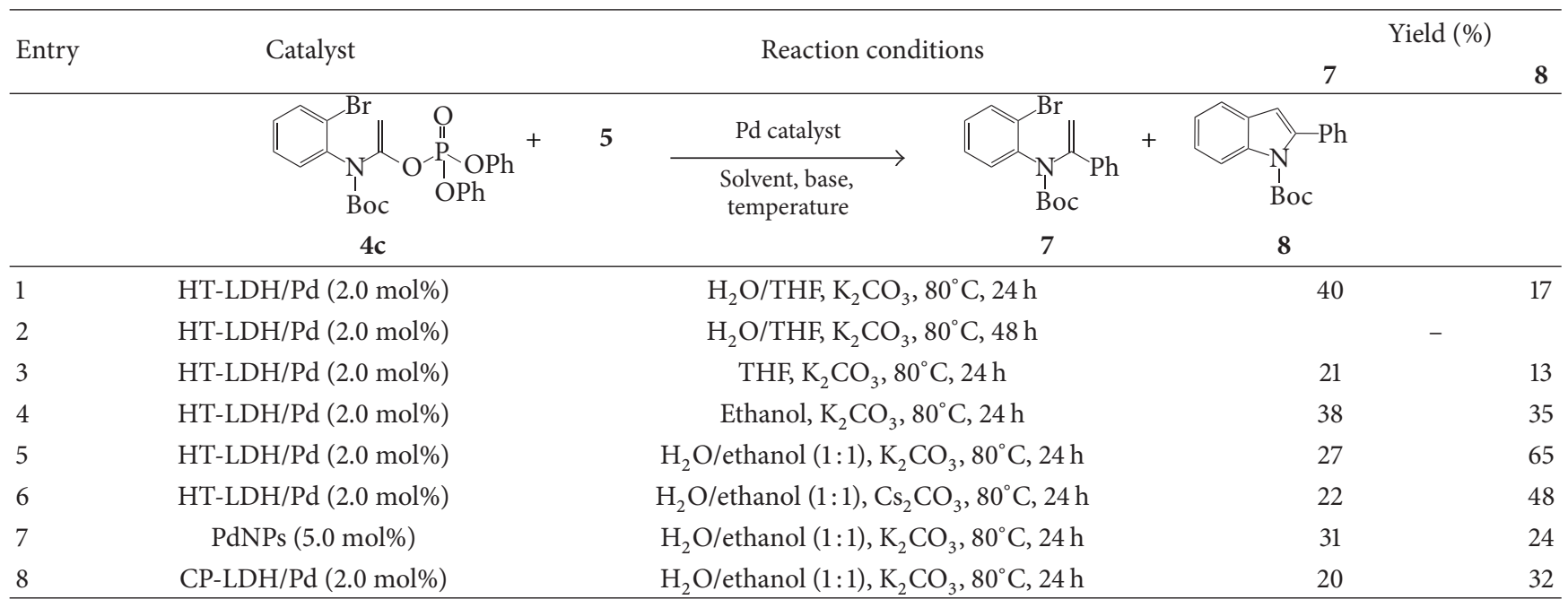

TABLE 3: Catalyst reusability ${ }^{1}$.

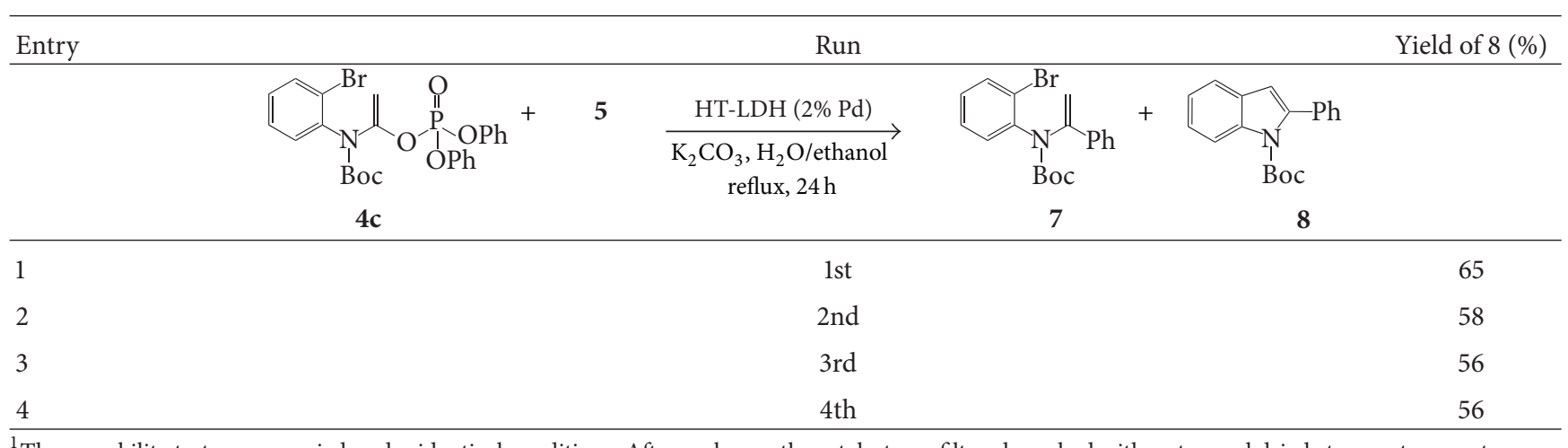

${ }^{1}$ The reusability tests were carried under identical conditions. After each run, the catalyst was filtered, washed with water, and dried at room temperature.

medium was filtered after the end of the reaction and the catalytic activity of the supernatant was analyzed under the same conditions. Interestingly, there was no detected amount of both coupling products, indicating that only a limited number of species were leached. Examination of the catalyst recyclability (Table 3 ) revealed that the material can be used for four consecutive runs without significant loss in activity. Based on these results, we hypothesized that the heterogeneous catalysis could be the predominant mechanism.

\section{Conclusion}

In summary, we have demonstrated that a catalyst system based on layered double hydroxide containing $\operatorname{Pd}(0)$ is efficient for the $\mathrm{C}-\mathrm{O}$ activation of acetamide-derived ketene aminal phosphates. Based on anilines containing Boc or $\mathrm{CBz}$ as protecting groups, we started catalytic studies for the crosscoupling reactions with the phenylboronic acid. The Boc substituted $o$-brominated enol phosphate was quite stable under the catalytic conditions when compared to the $\mathrm{CBz}$ counterpart evidencing that the combination between the electron-donating and electron-withdrawing groups involves a delicate balance. From the comparison with the classical catalytic conditions, the beneficial effect of this ligand-free strategy is apparent. By using the hydrothermally treated LDH containing $\operatorname{Pd}(0)$, the corresponding $\alpha$-aryl enecarbamates were obtained in moderate to good yields. In the case of the $o$-halo substituted substrate, the conditions were tuned to improve the domino Suzuki-Miyaura/intramolecular Heck which led to the 2-phenyl indole in moderate yields. Indeed, the catalyst showed recycling potential since the activity was similar for four consecutive runs towards the 2-phenyl indole. Although improvement of the scope of the methodology requires additional studies, the advantage of using a ligandfree condition, as in challenging $\mathrm{C}-\mathrm{O} / \mathrm{C}-\mathrm{C}$ domino reactions, is retained. Ongoing work seeks to explore additional substrates for these and related cross-coupling reactions.

\section{Supplementary Information}

${ }^{1} \mathrm{H}$ NMR spectra of all products are available online in the Supplementary Information file.

\section{Competing Interests}

The authors declare that they have no competing interests. 


\section{Acknowledgments}

The authors are grateful to FAPERJ, CAPES, and CNPq for financial support. They also would like to thank Bruno B. Lages for the graphical work in this manuscript and Laboratório Multiusuário de Difração de Raios-X at IQ/UFRJ for the XRD patterns.

\section{References}

[1] J. B. Pedley, R. D. Naylor, and S. P. Kirby, Thermochemical Data of Organic Compounds, Springer, Dordrecht, The Netherlands, 1986.

[2] J. D. Sellars and P. G. Steel, "Transition metal-catalysed crosscoupling reactions of P-activated enols," Chemical Society Reviews, vol. 40, no. 10, pp. 5170-5180, 2011.

[3] D.-G. Yu, B.-J. Li, and Z.-J. Shi, "Exploration of new C-O electrophiles in cross-coupling reactions," Accounts of Chemical Research, vol. 43, no. 12, pp. 1486-1495, 2010.

[4] B.-J. Li, D.-G. Yu, C.-L. Sun, and Z.-J. Shi, "Activation of "inert" alkenyl/aryl C-O bond and its application in cross-coupling reactions," Chemistry, vol. 17, no. 6, pp. 1728-1759, 2011.

[5] W. J. Scott, G. T. Crisp, and J. K. Stille, "Palladium-catalyzed coupling of vinyl triflates with organostannanes. A short synthesis of pleraplysillin-1," Journal of the American Chemical Society, vol. 106, no. 16, pp. 4630-4632, 1984.

[6] N. Jana, Q. Nguyen, and T. G. Driver, "Development of a Suzuki cross-coupling reaction between 2-azidoarylboronic pinacolate esters and vinyl triflates to enable the synthesis of [2,3]-fused indole heterocycles," Journal of Organic Chemistry, vol. 79, no. 6, pp. 2781-2791, 2014.

[7] N. H. Williams and P. Wyman, "Base catalysed phosphate diester hydrolysis," Chemical Communications, no. 14, pp. 12681269, 2001.

[8] R. Wolfenden, C. Ridgway, and G. Young, "Spontaneous hydrolysis of ionized phosphate monoesters and diesters and the proficiencies of phosphatases and phosphodiesterases as catalysts," Journal of the American Chemical Society, vol. 120, no. 4, pp. 833-834, 1998.

[9] Y. Tanigawa, K. Nishimura, A. Kawasaki, and S.-I. Murahashi, "Palladium(O)-catalyzed allylic alkylation and amination of allylic phosphates," Tetrahedron Letters, vol. 23, no. 52, pp. 55495552, 1982.

[10] S. Protti and M. Fagnoni, "Phosphate esters as "tunable" reagents in organic synthesis," Chemical Communications, no. 31, pp. 3611-3621, 2008.

[11] K. Takai, K. Oshima, and H. Nozaki, "Carboncarbon bond formation by cross-coupling of enol phosphates with organoaluminium compounds catalyzed by palladium $(\mathrm{O})$ complex," Tetrahedron Letters, vol. 21, no. 26, pp. 2531-2534, 1980.

[12] K. C. Nicolaou, G.-Q. Shi, J. L. Gunzner, P. Gartner, and Z. Yang, "Palladium-catalyzed functionalization of lactones via their cyclic ketene acetal phosphates. Efficient new synthetic technology for the construction of medium and large cyclic ethers," Journal of the American Chemical Society, vol. 119, no. 23, pp. 5467-5468, 1997.

[13] F. Lepifre, C. Buon, R. Rabot, P. Bouyssou, and G. Coudert, "Palladium-catalysed coupling of vinyl phosphates with aryl or heteroaryl boronic acids. Application to the synthesis of substituted nitrogen containing heterocycles," Tetrahedron Letters, vol. 40, no. 35, pp. 6373-6376, 1999.
[14] F. Lepifre, S. Clavier, P. Bouyssou, and G. Coudert, "Palladiumcatalyzed coupling of vinyl phosphates with aryl or heteroaryl boronic acids. Application to the synthesis of substituted nitrogen containing heterocycles," Tetrahedron, vol. 57, no. 32, pp. 6969-6975, 2001.

[15] J. Guo, J. D. Harling, P. G. Steel, and T. M. Woods, "Phosphinates as new electrophilic partners for cross-coupling reactions," Organic and Biomolecular Chemistry, vol. 6, no. 21, pp. 40534058, 2008.

[16] H. Fuwa, "Palladium-catalyzed synthesis of N- and Oheterocycles starting from enol phosphates," Synlett, no. 1, pp. 6-29, 2011.

[17] S. Begliomini, L. Sernissi, D. Scarpi, and E. G. Occhiato, "A short, chemo-enzymatic synthesis of both enantiomers of trans-3-hydroxypipecolic acid," European Journal of Organic Chemistry, vol. 2014, no. 25, pp. 5448-5455, 2014.

[18] H. Fuwa and M. Sasaki, "Strategies for the synthesis of 2substituted indoles and indolines starting from acyclic $\alpha$ phosphoryloxy enecarbamates," Organic Letters, vol. 9, no. 17, pp. 3347-3350, 2007.

[19] K. C. Nicolaou, G.-Q. Shi, K. Namoto, and F. Bernal, "Synthesis of $N$-heterocycles via lactam-derived ketene aminal phosphates. Asymmetric synthesis of cyclic amino acids," Chemical Communications, no. 16, pp. 1757-1758, 1998.

[20] A. B. C. Simas, D. L. de Sales, and K. C. Pais, "Acyclic ketene aminal phosphates derived from N,N-diprotected acetamides: stability and cross-couplings," Tetrahedron Letters, vol. 50, no. 50, pp. 6977-6980, 2009.

[21] G. Fan, F. Li, D. G. Evans, and X. Duan, "Catalytic applications of layered double hydroxides: recent advances and perspectives," Chemical Society Reviews, vol. 43, no. 20, pp. 7040-7066, 2014.

[22] A. D. C. Silva, A. L. F. De Souza, R. A. Simão, and L. F. Brum Malta, "A simple approach for the synthesis of gold nanoparticles mediated by layered double hydroxide," Journal of Nanomaterials, vol. 2013, Article ID 357069, 6 pages, 2013.

[23] A. D. C. Silva, J. D. Senra, A. L. F. de Souza, and L. F. Brum Malta, "A ternary catalytic system for the room temperature SuzukiMiyaura reaction in water," The Scientific World Journal, vol. 2013, Article ID 456789, 8 pages, 2013.

[24] S. Brunauer, P. H. Emmett, and E. J. Teller, "Adsorption of gases in multimolecular layers," Journal of the American Chemical Society, vol. 60, no. 2, pp. 309-319, 1938.

[25] M. Mora, C. Jiménez-Sanchidrián, and J. R. Ruiz, "Heterogeneous Suzuki cross-coupling reactions over palladium/hydrotalcite catalysts," Journal of Colloid and Interface Science, vol. 302, no. 2, pp. 568-575, 2006.

[26] M. Mora, M. I. Lopez, C. Jiménez-Sanchidrián, and J. R. Ruiz, "Near-infrared spectroscopy of palladium-containing layered double hydroxides used as catalysts," Journal of Physics and Chemistry of Solids, vol. 72, no. 3, pp. 214-219, 2011.

[27] G. R. Humphrey and J. T. Kuethe, "Practical methodologies for the synthesis of indoles," Chemical Reviews, vol. 106, no. 7, pp. 2875-2911, 2006.

[28] N. K. Garg, R. Sarpong, and B. M. Stoltz, "The first total synthesis of dragmacidin D," Journal of the American Chemical Society, vol. 124, no. 44, pp. 13179-13184, 2002.

[29] S. Cacchi and G. Fabrizi, "Synthesis and functionalization of indoles through palladium-catalyzed reactions," Chemical Reviews, vol. 105, no. 7, pp. 2873-2920, 2005.

[30] M. T. Hovey, C. T. Check, A. F. Sipher, and K. A. Scheidt, "N-heterocyclic-carbene-catalyzed synthesis of 2-aryl indoles," 
Angewandte Chemie-International Edition, vol. 53, no. 36, pp. 9603-9607, 2014.

[31] G. W. Gribble, Indole Ring Synthesis, John Wiley \& Sons, Chichester, UK, 2016.

[32] H. Fuwa and M. Sasaki, "Synthesis of 2-Substituted Indoles and Indolines via Suzuki-Miyaura Coupling/5-endo-trig Cyclization Strategies," Journal of Organic Chemistry, vol. 74, no. 1, pp. 212-221, 2009.

[33] J. A. Widegren and R. G. Finke, "A review of the problem of distinguishing true homogeneous catalysis from soluble or other metal-particle heterogeneous catalysis under reducing conditions," Journal of Molecular Catalysis A: Chemical, vol. 198, no. 1-2, pp. 317-341, 2003. 

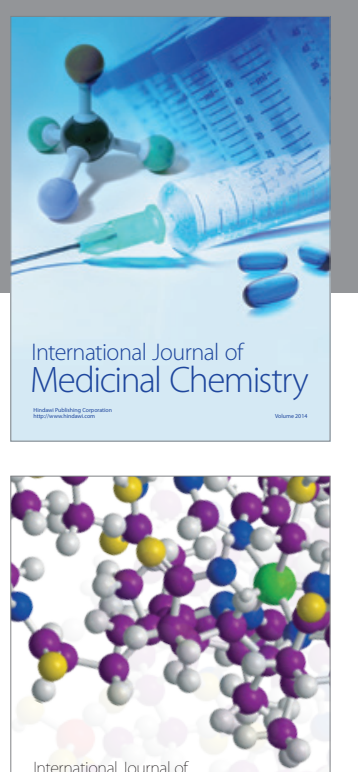

Carbohydrate Chemistry

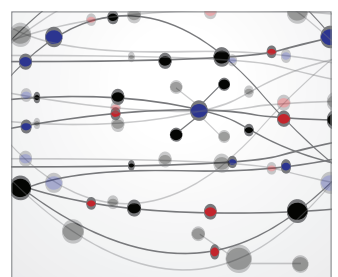

The Scientific World Journal
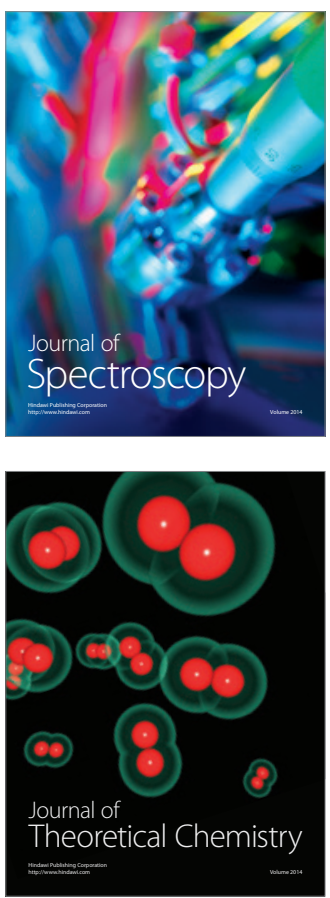
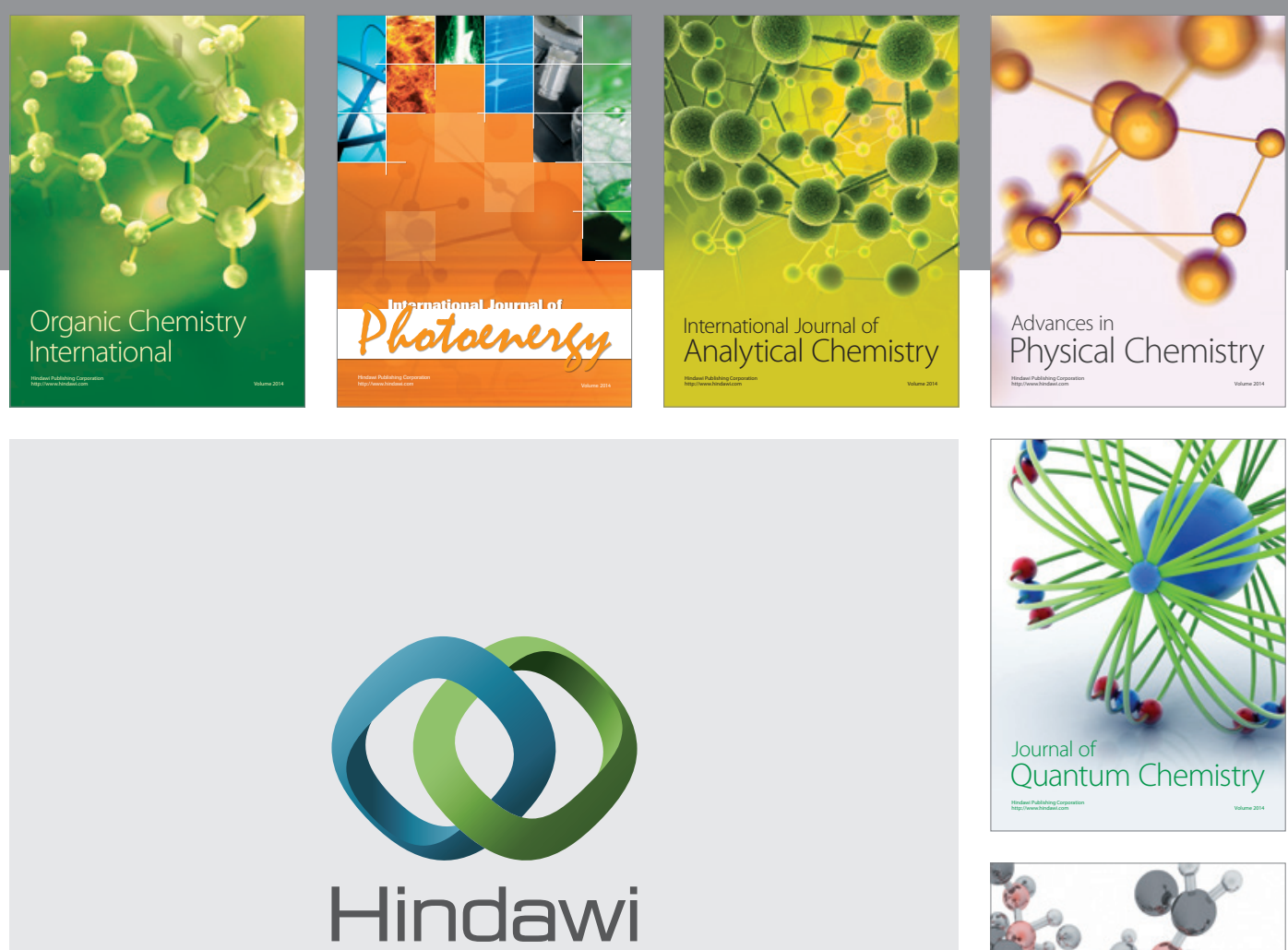

Submit your manuscripts at

https://www.hindawi.com

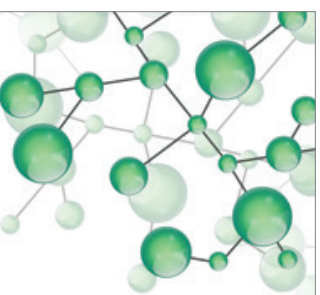

International Journal of

Inorganic Chemistry
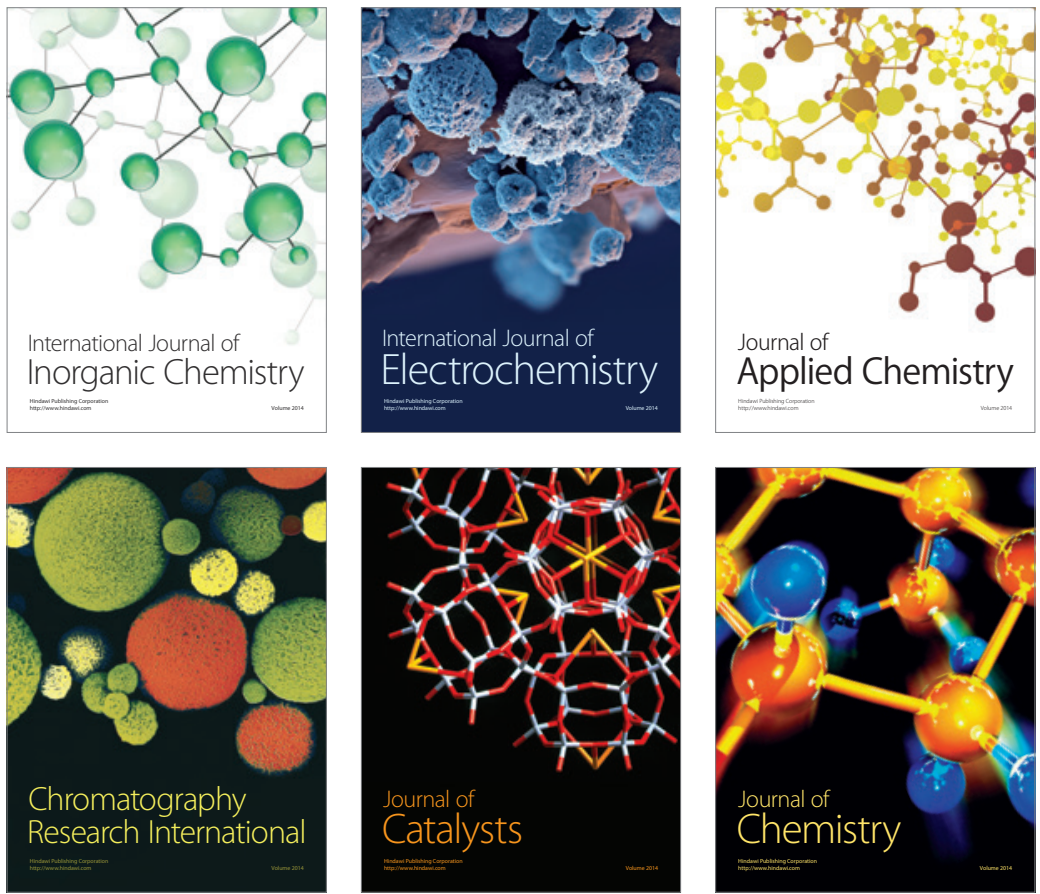

Journal of

Applied Chemistry
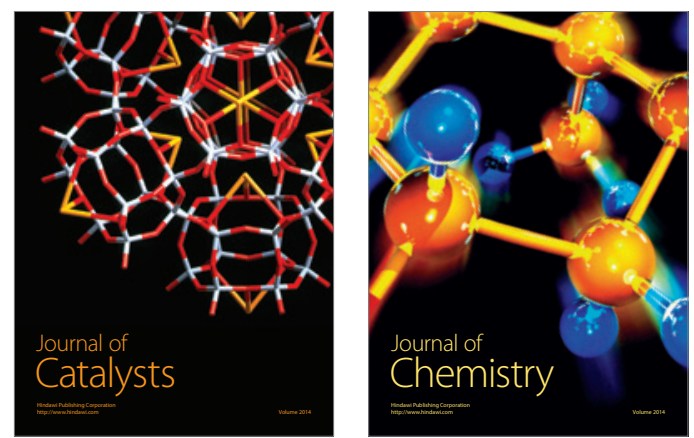
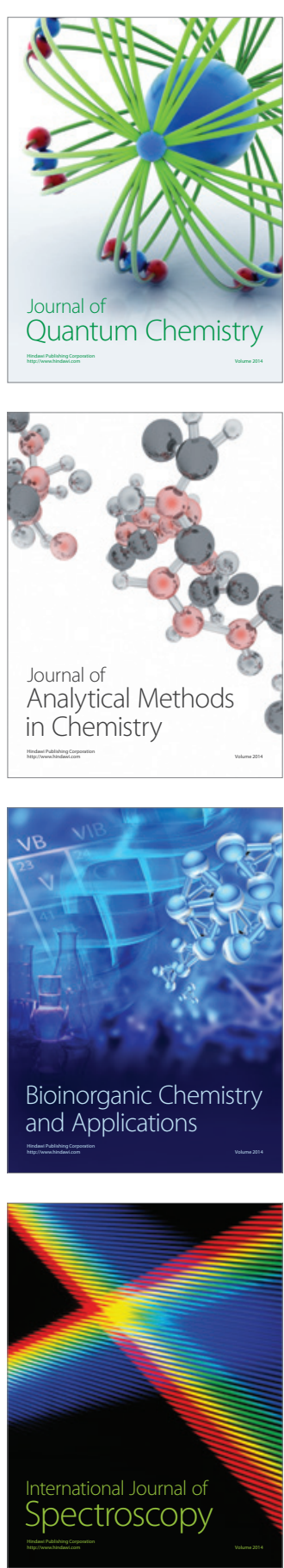